\title{
Sildenafil: efectos en la calidad espermática del hombre subfértil *
}

\author{
Dras. Elinor Virginia Moreno Acosta, ${ }^{1}$ Nellys Marina Fonseca Castillo, ${ }^{1}$ \\ Sheila Josefina Rodríguez Guevara. ${ }^{2}$
}

\begin{abstract}
RESUMEN
Objetivo: Evaluar el efecto del sildenafil en los parámetros espermáticos de hombres subfértiles que acudieron al servicio de fertilidad de la Maternidad Concepción Palacios entre junio 2011 y diciembre 2013.

Métodos: Se realizó un estudio clínico, prospectivo, descriptivo, experimental, de corte transversal, el cual se llevó a cabo previo consentimiento informado en 28 hombres subfértiles con astenozoospermia diagnosticada mediante la realización de dos espermogramas basales, posteriormente se les administró $50 \mathrm{mg}$ de sildenafil una hora antes de obtener la muestra para un tercer y último espermograma.

Resultados: Entre los 28 pacientes estudiados, se evidenció que después del tratamiento aumentó la motilidad progresiva en 21 pacientes (75\%) lo que resultó estadísticamente significativo $(p=0,000)$; el volumen eyaculado antes $y$ después del tratamiento no tuvo cambios estadisticamente significativos ( $p=0,405)$, al igual que la concentración espermática $(p=1,000)$.

Conclusiones: El uso del sildenafil en pacientes astenozoospérmicos representa una opción terapéutica económica, de fácil adquisición y cumplimiento en el tratamiento de dicha patología.
\end{abstract}

Palabras clave: Astenozoospermia, Sildenafil, Espermograma

\section{SUMMARY}

Objective: To evaluate the effect of sildenafil on the sperm parameters of subfertile men who attended the fertility service of the Maternidad Concepción Palacios, between June 2011 and December 2013.

Methods: A clinical, prospective, descriptive, experimental, cross-sectional study was conducted, prior informed consent, in 28 subfertile men with diagnosed asthenozoospermia, by performing two basal spermogram. Thereafter, they were given $50 \mathrm{mg}$ of sildenafil, one hour before obtaining the sample, for a third and final spermogram.

Results: Among the 28 patients studied, it was shown an increased progressive motility post-treatment in 21 patients (75\%) which was statistically significant ( $p-0.000)$; the volume ejaculated before and after treatment had no statistically significant differences (p-0.405), as well as sperm concentration ( $p$-1,000).

Conclusions: The use of sildenafil in patients with diagnosed asthenozoospermia represents an economical therapeutic option, easy to acquire, and comply with in the treatment of this pathology.

Keywords: Asthenozoospermia, Sildenafil, Semen.

\section{INTRODUCCIÓN}

${ }^{1}$ Especialistas en Obstetricia y Ginecología, egresadas del Programa de la Universidad Central de Venezuela, con sede en la Maternidad Concepción Palacios. ${ }^{2}$ Especialista en Obstetricia y Ginecología, exjefa de Servicio de Fertilidad, Maternidad Concepción Palacios.

*Trabajo Especial de Grado presentado y aprobado para optar al título de Especialista en Obstetricia y Ginecología.
La infertilidad masculina es producida por diferentes desórdenes que ameritan de una evaluación clínica integral. El advenimiento de técnicas de reproducción asistida, como opción para corregir el factor masculino grave ha hecho que estas se usen como primera línea de tratamiento, pero en muchos casos la terapia inicial 
debe dirigirse a mejorar la calidad del semen, para facilitar la obtención de un embarazo por medios naturales e, inclusive, el uso de tratamiento médico destinado a mejorar los componentes subcelulares del espermatozoide, puede mejorar los resultados de las técnicas de reproducción asistida (1).

La infertilidad masculina involucra alrededor de $35 \%$ a $45 \%$ de la casuística total de infertilidad. Esta puede deberse a causas congénitas, infecciosas, patología urológica, traumática, consecuencia de cirugía inguinoescrotal, asociada a enfermedades pulmonares crónicas, disfunción sexual, trastornos inmunológicos, genéticos, por lesiones neurológicas, por factores ambientales y tóxicos, tumorales o idiopáticas (2).

La administración del sildenafil aumenta la eficacia de los mecanismos fisiológicos que intervienen en la erección del pene en presencia de estímulo sexual (3). Su efecto en la motilidad del espermatozoide, está siendo estudiado por diversos investigadores.

El análisis seminal es una de las pruebas utilizadas en el diagnóstico de la infertilidad, entidad que afecta a un $10 \%-20 \%$ de las parejas en edad reproductiva. Puesto que en la mitad de los casos la causa de la infertilidad es de origen masculino, se hace imprescindible la adecuada realización y subsecuente interpretación de la información que se obtiene de esa prueba.

En el servicio de fertilidad de la Maternidad Concepción Palacios, las alteraciones de la función espermática tipo astenozoospermia ocupan un porcentaje importante dentro de las causas de infertilidad masculina allí identificadas, tomando en cuenta que los tratamientos para dicha patología son de alto costo y complejidad y, conociendo que existen a nivel internacional trabajos realizados que suponen que el uso de sildenafil en hombres subfértiles mejora la motilidad del espermatozoide y de esta manera puede contribuir a lograr la concepción, surgió la idea de realizar este trabajo para evaluar el efecto del fármaco en la población masculina subfértil que acudió a la institución en un periodo de dos años.

Es poca la literatura que aporta datos acerca del efecto del sildenafil en la motilidad espermática, internacionalmente existen diferentestrabajosque tratan del tema; Aversa y cols. (4), en 2000, concluyeron que en pacientes con fertilidad probada, el tratamiento con sildenafil no causa cambios significativos en el número de espermatozoides y en la motilidad espermática. La óxido nítrico sintetasa y dos isoformas diferentes de fosfodiesterasa (PDE 1 y PDE 4) están presentes en los espermatozoides humanos. El sildenafil es un inhibidor potente y específico de la fosfodiesterasa tipo 5 GMPc específica, que es la isoenzima responsable de la degradación del GMPc en el cuerpo cavernoso, y tiene efectos inhibitorios de menor importancia en las actividades de la PDE 6 y 1 . Concluyen que debido a que las mejoras en los parámetros seminales de estos inhibidores de la PDE son menores, los efectos del sildenafil en hombres oligozoospérmicos pueden ser más pronunciados y debe ser evaluado en un futuro. Por su parte, Lefievre y cols. (5) para el mismo año, determinaron que el tratamiento oral con sildenafil estimula la motilidad espermática, probablemente por su acción inhibitoria sobre la fosfodiesterasa tipo 5, incrementando el AMPc, resultados similares fueron obtenidos por Plesis y cols. (6) y Jannini y cols (7), en 2004, y Mostafa y cols. (8), en 2007. Burger y cols. (9) encontraron que el sildenafil no tuvo ningún efecto estadísticamente significativo sobre la motilidad del esperma, el recuento o la densidad, el porcentaje de formas anormales de espermatozoides o el porcentaje de la esperma. Así mismo, no afectó el volumen eyaculado o la viscosidad. Todas las medidas se mantuvieron dentro de rangos normales, después de su administración en hombres normales e infértiles. Pomara y cols. (10) indicaron que la motilidad de los espermatozoides parece estar muy afectada en los pacientes jóvenes infértiles con una única dosis de sildenafil y tadalafilo, con efectos opuestos: estimulante primero e inhibidor después. Glenn y 
cols. (11) demostraron que tanto el número como la velocidad de motilidad progresiva del espermatozoide se incrementó de manera significativa con el citrato del sildenafil, pero, controversialmente, este también aumentó de forma importante la proporción de reacción acrosómica del espermatozoide. Finalmente, Boorjian y cols. (12) identificaron la naturaleza de la disfunción sexual asociada con la infertilidad de factor masculino, y demostraron la eficacia de la terapia de sildenafil en hombres con esta condición.

Aproximadamente $85 \%$ de las parejas son capaces de concebir después de un año de relaciones sin protección, el $15 \%$ no lo logra. En aproximadamente $30 \%$ de estas, el factor masculino es responsable por si solo y en $20 \%$ existe combinación de ambos, por lo tanto se dice que el factor masculino está involucrado en casi la mitad de los casos que consultan por problemas de infertilidad (13).

El manejo de estos casos debe iniciarse con la historia clínica y el examen físico seguido por el laboratorio (13). La historia clínica debe detallar aspectos como la edad, los hábitos sexuales, enfermedades de la infancia en el hombre, como la criptorquidia uni- o bilateral, historia de traumatismo testicular y/o torsión testicular, historia de cirugías previas en vejiga, pelvis, región inguinal o retroperitoneo, antecedentes de parotiditis y la orquitis urliana, infecciones del tracto genitourinario, cualquier estado de fiebre o viremia que pueda afectar la espermatogénesis, la exposición a químicos y drogas, factores de la vida cotidiana que afectan la calidad del semen como cigarrillos, alcohol, estrés y aumento de la temperatura. Los aspectos nutricionales también se han visto relacionados con la función espermatogénica. (13)

El examen físico debe ser completo, con especial énfasis en el área genital; los testículos y la exploración peritesticular es fundamental (13).
Respecto a las pruebas de laboratorio, el espermatograma consiste en el análisis macro y microscópico del semen, recolectado en forma adecuada, haciendo hincapié en el tiempo y lugar de recolección. Generalmente, se recomiendan cinco días de abstinencia y la forma de recolección más recomendada es la masturbación; sin embargo, el coitus interruptus o el uso de preservativos libres de sustancias tóxicas son válidos. El recipiente debe estar limpio, no necesariamente estéril (14). Los valores que se evalúan en el espermatograma se presentan en el cuadro 1 y los valores mínimos para considerar un espermatograma normal según la Organización Mundial de la Salud (OMS) para el 2010 (al menos en dos oportunidades) se presentan en el cuadro 2.

El espermatograma puede revelar:

- Azoospermia: ausencia de espermatozoides.

- Oligospermia: menos de 15 millones de espermatozoides $/ \mathrm{ml}$. Se considera severa si hay menos de 10 millones $/ \mathrm{ml}$.

- Astenozoospermia: menos del $32 \%$ de la motilidad progresiva.

- Teratozoospermia: menos del $4 \%$ de formas normales.

- Oligoteratoastenozoospermia: combinación de las 3 alteraciones.

La calidad espermática es considerada clásicamente en términos de la movilidad como la morfología y la vitalidad (14).

La disminución del valor absoluto de los espermatozoides con movilidad progresiva puede deberse a alteraciones en la ultraestructura del flagelo o bien a trastornos producidos cuando el espermatozoide entra en contacto con las secreciones de las glándulas 
Cuadro 1. Valores que se evalúan en el espermatograma (14)

\begin{tabular}{ll}
\hline $\begin{array}{l}\text { Características de plasma } \\
\text { seminal }\end{array}$ & $\begin{array}{l}\text { Biofísicas (volumen, viscosidad). } \\
\text { Bioquímicas (pH, marcadores glandulares: fructuosa, alfaglucosidasa). } \\
\text { Concentración, movilidad, morfología (criterios estrictos). }\end{array}$ \\
$\begin{array}{l}\text { Características de los } \\
\text { espermatozoides }\end{array}$ & $\begin{array}{l}\text { Vitalidad (coloraciones vitales, prueba de hinchamiento espermático HOST). } \\
\text { Separación de fracción móvil tipo alfa. }\end{array}$ \\
$\begin{array}{l}\text { Identificación de células } \\
\text { seminales }\end{array}$ & $\begin{array}{l}\text { Leucocitos seminales. } \\
\text { Células germinales inmaduras }\end{array}$ \\
Inmunológicas & Anticuerpos antiespermáticos. \\
Microbiológicas & Cultivo seminal. Anticuerpos anti Chlamydia Trachomatis. \\
Funcionalidad de los & $\begin{array}{l}\text { Reacción acrosómica, unión a la zona pelúcida, penetración espermática. } \\
\text { espermatozoides }\end{array}$ \\
\hline
\end{tabular}

Cuadro 2. Valores mínimos para considerar un espermatograma normal (14)

\begin{tabular}{lc}
\hline Parámetros & Valores normales \\
\hline Volumen & $1,5 \mathrm{ml}(1,4-1,7 \mathrm{ml})$ \\
Número total de & 39000000 \\
espermatozoides & $(33$ a 46 millones $)$ \\
Concentración de & $15000000 / \mathrm{ml}$ \\
espermatozoides & $(10$ a 12 millones $/ \mathrm{ml})$ \\
Motilidad total & Mayor a $40 \%$ \\
(PR + NP) & $(38 \%-42 \%)$ \\
Motilidad progresiva & $32 \%(31 \%-34 \%)$ \\
Vitalidad & $58 \%(55 \%-63 \%)$ \\
Morfología & $4 \%(3-4)$. \\
Otros valores del consenso & \\
pH & $\geq 7,2$ \\
Leucocitos peroxidasa- & $<1000000$ \\
positivo & $<50 \%$ \\
MAR test & $<50 \%$ \\
Inmunobead test & $\geq 2,4 \mu \mathrm{mol}$ \\
Zinc & $\geq 13 \mu \mathrm{mol}$ \\
Fructuosa & $\geq 20 \mu \mathrm{mol}$ \\
$\alpha$-glucosidasa & \\
\hline
\end{tabular}

del tracto masculino. Otras causas de disminución de la movilidad son los defectos citogenéticos, la presencia de anticuerpos antiespermáticos, alteraciones en la morfología espermática, varicocele e infecciones de las vías seminales (14).

Según la Organización Mundial de la Salud (OMS), la motilidad espermática se clasifica en progresiva rápida, progresiva moderada, lenta e inmóvil. En una gota de la muestra se cuentan cien espermatozoides, que se clasifican según su movilidad. Se considera motilidad normal cuando la suma de los progresivos rápidos más los progresivos moderados es mayor o igual al $32 \%$. Cuando es menor al $32 \%$ se denomina astenozoospermia. (14).

El sildenafil es un potente inhibidor selectivo de la fosfodiesterasa tipo 5 (FDE-5), guanosinamonofosfato cíclico (GMP cíclico) específica. La respuesta del tejido eréctil del pene es mediada por el óxido nítrico $(\mathrm{ON})$ liberado por acción de las neuronas y las células endoteliales del pene durante el estímulo sexual. El ON a través de la activación enzimática de la guanilato-ciclasa, estimula la síntesis del mensajero GMP cíclico, la que a su vez resulta en una cascada de eventos que finaliza con la relajación del músculo liso de los cuerpos cavernosos y un mayor ingreso 
de sangre a los mismos, que inicia el proceso de tumescencia peneana. Esta alcanza su máximo cuando, por compresión, se produce la oclusión del retorno venoso. La inhibición de la actividad FDE-5 por efecto de sildenafil, impide la hidrólisis del GMP cíclico y por consiguiente obtiene una mayor concentración intracelular de este mensajero y una mayor y duradera erección. En los niveles de dosis recomendados, se han observado efectos del sildenafil solo en presencia de estimulación sexual. La importancia de la alta selectividad de acción sobre la FDE-5 en relación con otras FDE, reside en la ausencia de efectos del sildenafil, en voluntarios sanos, sobre la contracción cardiaca in vitro y el electrocardiograma. Su menor selectividad con respecto a la FDE-6, que se encuentra principalmente en retina, explica algunos trastornos visuales observados con las dosis más altas, que los pacientes describen como sensibilidad a la luz o visión teñida de color (3). Las contraindicaciones para su uso son hipersensibilidad conocida al sildenafil o a alguno de los componentes del producto. Pacientes en tratamiento con nitratos orgánicos o dadores de óxido nítrico. Antes de indicar el tratamiento con el sildenafil el paciente debe ser evaluado exhaustivamente para determinar la etiología de la disfunción eréctil. Sildenafil debe ser administrado con precaución en pacientes con anormalidades anatómicas del pene como angulación, fibrosis cavernosa (enfermedad de Peyronie) y en aquellos proclives a presentar priapismo u otros trastornos como anemia de células falciformes, mieloma múltiple o leucemia. También en los pacientes con riesgo de hemorragia o úlcera gastroduodenal activa. Dado que la actividad sexual puede incrementar el riesgo de algunos trastornos cardiacos, es conveniente evaluar la función cardiovascular antes de instaurar el tratamiento. El sildenafil debe ser administrado con cautela en los pacientes con retinitis pigmentaria debido a que en algunos individuos que padecen esta patología existe un trastorno genético en las fosfodiesterasas de la retina. El sildenafil puede administrarse con otros medicamentos que son utilizados para tratar padecimientos relacionados con la disfunción eréctil como son los hormonales o que incluso pueden provocar directamente disfunción eréctil, como son algunos agentes antihipertensivos (3).

La presente investigación se realizó con el objetivo de evaluar el efecto del sildenafil en los parámetros espermáticos de hombres subfértiles.

\section{MÉTODOS}

Se trató de un estudio clínico, prospectivo, descriptivo experimental, transversal. Se calculó una muestra probabilística empleando una proporción de $0,135 \%$ (porcentaje de hombres subfértiles con astenozoospermia según la morbilidad del servicio). Se obtuvo una muestra de 28 pacientes que llenaran los siguientes criterios: hombres entre 18 y 50 años, con 1 año o más de relaciones con una pareja estable, sin lograr embarazo y cuyo espermograma (por lo menos dos) evidenciaron astenozoospermia. Se excluyeron pacientes diabéticos no controlados con retinopatía diabética asociada, enfermedad vascular preexistente (estenosis aórtica, estenosis subaórtica, hipertrófica idiopática), historia previa de infarto de miocardio, arritmias severas, trastornos endocrinos, tratamiento con nitritos y anticoagulantes, signos de infección espermática, deformidades anatómicas de los genitales que perjudiquen la erección, niveles elevados de prolactina y niveles bajos de testosterona, hipotensión ( $\mathrm{TA}<90 / 50 \mathrm{mmHg}$ ), hipertensión ( $\mathrm{TA}>170 / 110 \mathrm{mmHg}$ ), historia de retinitis pigmentosa, alergia al sildenafil.

Todos los pacientes firmaron un formulario de consentimiento informado.

A los pacientes que llenaron los criterios, se les administró una dosis vía oral de $50 \mathrm{mg}$ de sildenafil, una hora después se les solicitó que recolectaran una muestra para un tercer y último espermograma. 
Se calcularon las frecuencias y porcentajes de las variables nominales. Los contrastes entre las variables entre el basal y postratamiento, se basaron en la prueba de McNemar (respuestas dicotómicas) y en la prueba de homogeneidad marginal (respuestas con más de dos categorías). Se consideró un valor estadísticamente significativo si $\mathrm{p}<0,05$. Los datos fueron analizados con JMP-SAS versión 11.0.

\section{RESULTADOS}

Se incluyeron 28 pacientes astenozoospérmicos, de ellos $6(21,4 \%)$ tenían además oligozoospermia (tabla 1$)$.

En la tabla 2 se observa que en 25 de los 28 pacientes $(89,3 \%)$ la motilidad progresiva era menor del $31 \%$ y en $3(10,7 \%)$ estaba entre $31 \%$ y $34 \%$. Una hora después de la toma del sildenafil, 21 pacientes $(75 \%)$ tenían una motilidad progresiva mayor de $34 \%(\mathrm{p}=0,000)$.

El volumen del eyaculado representado en la tabla 3, fue mayor de $1,7 \mathrm{ml}$, en la mayoría de los pacientes tanto en los espermogramas basales como postratamiento $(\mathrm{p}=0,405)$.

Los 28 pacientes tuvieron un $\mathrm{pH}$ mayor o igual a 7,2 en ambas muestras (basal y postratamiento) ( $\mathrm{p}$ de $\mathrm{McNemar}=1,000)$. En relación a la morfología, todos los pacientes tenían $4 \%$ o más de formas normales de espermatozoides en la prueba basal; postratamiento 23 pacientes $(82,1 \%)$ tenían $4 \%$ o más, y $4(14,3 \%)$ tenían menos del $4 \%(\mathrm{p}=0,125)$.

Tabla 1. Distribución de pacientes según las alteraciones del espermograma de acuerdo a la nomenclatura de la OMS.

\begin{tabular}{lcc}
\hline Alteraciones & $\mathrm{n}$ & $\%$ \\
\hline Oligozoospermia & 6 & 21,42 \\
Astenozoospermia & 28 & 100 \\
\hline
\end{tabular}

Tabla 2. Distribución de pacientes según la motilidad progresiva de acuerdo a la OMS.

\begin{tabular}{lcccc}
\hline \multirow{2}{*}{$\begin{array}{l}\text { Motilidad } \\
\text { progresiva }\end{array}$} & \multicolumn{2}{c}{ Basal } & \multicolumn{2}{c}{ Postratamiento } \\
\cline { 2 - 5 } & $\mathrm{n}$ & $\%$ & $\mathrm{n}$ & $\%$ \\
\hline Menor de 31\% & 25 & 89,28 & 3 & 10,71 \\
$31 \%-34 \%$ & 3 & 10,71 & 4 & 14,28 \\
Mayor de 34 \% & 0 & 0 & 21 & 75 \\
\hline & \multicolumn{3}{c}{$\mathrm{Z}=4,703(\mathrm{p}=0,000)$}
\end{tabular}

Tabla 3. Distribución de pacientes según el volumen del eyaculado de acuerdo a la OMS

\begin{tabular}{lcccc}
\hline \multirow{2}{*}{ Volumen } & \multicolumn{2}{c}{ Basal } & \multicolumn{2}{c}{ Postratamiento } \\
\cline { 2 - 5 } & $\mathrm{n}$ & $\%$ & $\mathrm{n}$ & $\%$ \\
\hline Menor de $1,4 \mathrm{ml}$ & 0 & 0 & 2 & 7,14 \\
$1,4 \mathrm{ml}-1,7 \mathrm{ml}$ & 3 & 10,71 & 2 & 7,14 \\
Mayor de $1,7 \mathrm{ml}$ & 25 & 89,28 & 24 & 85,71 \\
\hline & & \multicolumn{3}{c}{$\mathrm{Z}=0,832(\mathrm{p}=0,405)$}
\end{tabular}

En la tabla 4 se observó que en el espermograma basal en 22 pacientes $(78,6 \%)$, la concentración espermática fue mayor o igual a 15 millones de espermatozoides por $\mathrm{ml}$. En el postratamiento, 21 pacientes tenían concentración mayor o igual a 15 millones (75\%), en un caso no se reportó la concentración ( $\mathrm{p}$ de McNemar=1,000).

Tabla 4. Distribución de pacientes según la concentración espermática de acuerdo a la OMS

\begin{tabular}{lcccc}
\hline \multirow{2}{*}{$\begin{array}{l}\text { Millones de } \\
\text { espermatozoides / ml }\end{array}$} & \multicolumn{2}{c}{ Basal } & \multicolumn{2}{c}{ Postratamiento } \\
\cline { 2 - 5 } & $\mathrm{n}$ & $\%$ & $\mathrm{n}$ & $\%$ \\
\hline Mayor o igual a 15 & 22 & 78,57 & 21 & 75 \\
Menor de 15 & 6 & 21,42 & 6 & 21,42 \\
\hline
\end{tabular}

$\mathrm{p}$ de $\mathrm{McNemar}=1,000$ 


\section{DISCUSIÓN}

El estudio de la pareja infértil se ha convertido en un procedimiento de gran importancia para el médico de atención primaria quien, en la mayoría de los casos, establece el primer contacto con las parejas que tienen dificultad para salir embarazadas. La etiología de la infertilidad varía mucho con la población estudiada, nivel social, raza, si la atención que se brinda es primaria o avanzada, etc.; en general, se considera que las tres causas más frecuentes son las alteraciones espermáticas, las ovulatorias y las enfermedades tuboperitoneales (15).

La responsabilidad masculina en la falta de procreación de la pareja infértil fue negada durante muchos siglos. Aun hoy en día, debido a que es en la mujer en quien se desarrolla el embarazo, persiste en algunos estratos sociales el mito de la sola responsabilidad femenina (16). Estudios de la OMS indican que en parejas infértiles, el $35 \%$ de las mujeres tienen "sin causa aparente" como diagnóstico final de su infertilidad; al evaluar al hombre, esta cifra se eleva a un $47 \%$. El adecuado diagnóstico de las causas de infertilidad en el varón dependerá, en parte, del entendimiento de la regulación de la función espermática (17).

La astenozoospermia o astenospermia es la disminución de la movilidad de los espermatozoides del hombre y compromete la fertilidad debido a que impide que estos se desplacen hasta el óvulo y lo penetren (18). La astenozoospermia o motilidad espermática reducida puede ser aislada o combinada con oligospermia u otros parámetros anormales del semen (19). En la presente serie se incluyeron 28 pacientes que tenían diagnóstico de astenozoospermia, de los cuales 6 presentaban además oligozoospermia.

El análisis del semen es muy importante en la valoración de la fertilidad masculina (19). Comprende la evaluación de sus características fisicoquímicas (volumen, $\mathrm{pH}$, viscosidad, aglutinación), aspectos citomorfológicos (concentración y grado de movilidad espermática, morfología, leucocistospermia), aspectos funcionales, presencia de anticuerpos antiespermáticos, estudios microbiológicos y de marcadores glandulares (14).

La OMS desarrolló una serie de criterios de normalidad espermática para el año 2010 (cuadro 2), los cuales fueron utilizados en esta investigación y, con base en ellos, se evidenció que todos los pacientes objeto de estudio presentaron volumen, $\mathrm{pH}$, morfología y concentración espermática normal, en la prueba basal y postratamiento.

Morales y cols. (20) mostraron una tendencia positiva a mejorar la motilidad espermática total en pacientes astenozoospérmicos después de la administración de $100 \mathrm{mg}$ de sildenafil. De manera similar, en este trabajo se evidenció que el sildenafil administrado una hora antes del eyaculado en pacientes subfértiles con diagnóstico de astenozoospermia produce un aumento significativo de la motilidad progresiva, lo que se observó en el $75 \%$ de los pacientes, es decir, las tres cuartas partes de la población. Se destaca el hecho que en la presente serie se utilizó una dosis de $50 \mathrm{mg}$, inferior a la descrita por Morales y cols. (20), pero con iguales resultados.

El uso del sildenafil, aparte de ser una gran ayuda para solventar los problemas de erección, ha demostrado ser eficaz en casos de infertilidad. Si el hombre con disfunción eréctil y problemas de fertilidad lo que desea es tener hijos y lograr eyacular un semen normal, puede lograr no solo tener una relación sexual satisfactoria sino procrear (21).

Si bien es cierto que las técnicas de reproducción asistida, como la microinyección intracitoplasmática de espermatozoides (ICSI), son opciones para corregir el factor masculino grave y la infertilidad masculina 
idiopática, en muchos casos la terapia inicial debe dirigirse a mejorar la calidad del semen para facilitar la obtención de un embarazo por medios naturales. Además, estas terapias mejoran los componentes subcelulares del espermatozoide, lo que puede potenciar los resultados de las técnicas de reproducción asistida (1).

Los resultados del presente estudio permiten concluir que el sildenafil es eficaz para mejorar la motilidad espermática en pacientes subfértiles, sin afectar los otros parámetros del espermograma.

En vista de los resultados satisfactorios obtenidos con el uso del sildenafil en el presente estudio, este se ofrece como una alternativa terapéutica accesible, de bajo costo y fácil cumplimiento para pacientes subfértiles, siempre y cuando no este contraindicado.

Las autoras desean agradecer a la asesora metodológica, Dra. Mireya González Blanco; a los asesores estadísticos, Dr. Freddy Bello y Lic. Douglas Angulo; a la Dirección del Hospital y a la Dra. Ma. Teresa Urbina y demás personal del laboratorio de Unifertes quienes canalizaron el procesamiento de todas las muestras; al servicio de fertilidad de la Maternidad Concepción Palacios; a laboratorios VALMORCA, por suministrar el fármaco utilizado y a la biblioteca de la Sociedad Venezolana de Obstetricia y Ginecología.

\section{REFERENCIAS}

1. Teppa A, Palacios A. Tratamiento convencional y avanzado de la infertilidad masculina. Rev Reprod Hum. 2003: 32-40.

2. Teppa A, Palacios A. Evaluación actual de la infertilidad masculina. Invest Clín [Internet]. 2004 [consultado diciembre 2012]; 45 (4): 355-370. Disponible en: $\quad$ http://ve.scielo.org/scielo.php?script=sci arttext\&pid=S0535-51332004000400008

3. Dehesa M, Gómez L, Medina J, Mora J, Obadía L,
Quinzaños F, et al. Ocho años de experiencia con Sildenafil. Una revisión sobre eficacia y seguridad. Rev Mex Urol [Internet]. 2007 [consultado diciembre 2012]; 67(1):35-48. Disponible en: https://www.medigraphic. com/pdfs/uro/ur-2007/ur071e.pdf

4. Aversa A, Mazzilli F, Rossi T, Delfino M, Isidori AM, Fabbri A. Effects of sildenafil (Viagra) administration on seminal parameters and post-ejaculatory refractory time in normal males. Hum Reprod. 2000; 15(1):131-4. doi: 10.1093/humrep/15.1.131. PMID: 10611201.

5. Lefièvre L, De Lamirande E, Gagnon C. The cyclic GMP-specific phosphodiesterase inhibitor, sildenafil, stimulates human sperm motility and capacitation but not acrosome reaction. J Androl [Internet]. 2000 [consultado noviembre 2012]; 21(6):929937. Disponible en: https://pubmed.ncbi.nlm.nih. gov/11105920/

6. du Plessis SS, de Jongh PS, Franken DR. Effect of acute in vivo sildenafil citrate and in vitro 8-bromo-cGMP treatments on semen parameters and sperm function. Fertil Steril. 2004; 81(4):1026-33. doi: 10.1016/j. fertnstert.2003.09.054. PMID: 15066459.

7. Jannini EA, Lombardo F, Salacone P, Gandini L, Lenzi A. Treatment of sexual dysfunctions secondary to male infertility with sildenafil citrate. Fertil Steril. 2004; 81(3):705-7. doi: 10.1016/j.fertnstert.2003.08.032. PMID: 15037428.

8. Mostafa T. In vitro sildenafil citrate use as a sperm motility stimulant. Fertil Steril. 2007; 88(4):994-6. doi: 10.1016/j.fertnstert.2006.11.182. Epub 2007 Feb 20. PMID: 17316632.

9. Burger M, Sikka SC, Bivalacqua TJ, Lamb DJ, Hellstrom WJ. The effect of sildenafil on human sperm motion and function from normal and infertile men. Int J Impot Res. 2000; 12(4):229-34. doi: 10.1038/ sj.ijir.3900551. PMID: 11079364.

10. Pomara G, Morelli G, Canale D, Turchi P, Caglieresi C, Moschini C, Liguori G, Selli C, Macchia E, Martino E, Francesca F. Alterations in sperm motility after acute oral administration of sildenafil or tadalafil in young, infertile men. Fertil Steril. 2007; 88(4):860-5. doi: 10.1016/j.fertnstert.2006.12.019. Epub 2007 Jun 4. PMID: 17544419.

11. Glenn DR, McVicar CM, McClure N, Lewis SE. Sildenafil citrate improves sperm motility but causes a premature acrosome reaction in vitro. Fertil Steril. 2007; 
87(5):1064-70. doi: 10.1016/j.fertnstert.2006.11.017. Epub 2007 Feb 28. PMID: 17335822.

12. Boorjian S, Hopps CV, Ghaly SW, Parker M, Mulhall JP. The utility of sildenafil citrate for infertile men with sexual dysfunction: a pilot study. BJU Int. 2007; 100(3):603-6. doi: 10.1111/j.1464-410X.2007.07038.x. Epub 2007 Jul 21. PMID: 17590181.

13. Narváez H. Actualización en la valoración y manejo de la infertilidad masculina. Rev Col Obstet Ginecol [Internet]. 2004 [consultado agosto 2013]; 55(1): 6070. Disponible en: http://www.scielo.org.co/pdf/rcog/ v55n1/v55n1a08.pdf

14. Urbina M, Lerner J. Fertilidad y reproducción asistida. Caracas: Editorial Médica Panamericana; 2008.

15. Pagés G, Aller J. Infertilidad. Caracas: Editorial Amolca; 2006.

16. Zighelboim I. Actualidades en reproducción humana y perinatología: Homenaje al Dr. Oscar Agüero. Caracas: Ediciones Lerner Internacional; 1982.

17. Rodríguez O, Acosta A, Silva H. Avances en medicina reproductiva. Caracas: Ediciones galénicas; 1993.

18. Instituto Ingenes. Fertilidad y Genética [Internet]. México: Astenozoospermia; 2011 [consultado agosto 2013]. Disponible en: http://www.ingenes.com/ primeros-pasos/entendiendo-la-infertilidad/causas/ factor-masculino/astenozoospermia/
19. Schorge J, Schaffer J, Halvorson L, Hoffman B, Bradshaw K, Cunningham F, editores. Williams Ginecología. Ciudad de México: Mc Graw-Hill Interamericana S.A; 2009.

20. Morales F, Merino M, Angulo E, Luna H, Guerrero G, Sordia L. Efectos del sildenafil sobre la motilidad espermática en pacientes con astenozoospermia [Internet]. Monterrey: XXVI Congreso Nacional de Investigación en Medicina; 2011 [consultado diciembre 2012]. Disponible en: http://www.medicina.uanl.mx/ congreso/memorias $2011 / \mathrm{htm} / 1560 / 648$.htm

21. Informamos.net [Internet]. Bogotá: La infertilidad masculina tiene que ver con la disfunción sexual. Cerca de 152 millones de hombres han experimentado en algún momento de su vida la disfunción eréctil; 2013 [consultado agosto de 2013]. Disponible en: http:// www.informamos.net/030615/infertilidad.htm

Recibido 17 de julio de 2020 Aprobado 14 de septiembre de 2020 